\title{
Association of TNFAIP3 gene polymorphism (rs5029939) with susceptibility and clinical phenotype of systemic lupus erythematosus
}

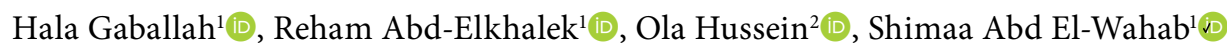 \\ ${ }^{1}$ Department of Rheumatology and Rehabilitation, Faculty of Medicine, Zagazig University, Zagazig, Egypt \\ ${ }^{2}$ Department of Clinical Pathology, Faculty of Medicine, Zagazig University, Zagazig , Egypt
}

\begin{abstract}
Objectives: This study aims to investigate the association of the tumor necrosis factor-alpha inducible protein 3 (TNFAIP3) (rs5029939) gene single nucleotide polymorphism (SNP) with the risk of systemic lupus erythematosus (SLE) and its clinical manifestations in a cohort of SLE patients.

Patients and methods: This study included a total of 180 participants (18 males, 72 females; mean age: $30.9 \pm 10.1$ years; range 17 to 59 years) including 90 SLE patients and 90 healthy controls between March 2017 and February 2020. The TNFAIP3 rs5029939 gene polymorphism was identified by polymerase chain reaction-restriction fragment length polymorphism (PCR-RFLP) in all participants.

Results: There was a significant difference in genotype distribution of the TNFAIP3 rs5029939 SNP between SLE patients and healthy controls, where CG genotype was more common in SLE patients $(53.3 \%)$ than controls $(11.1 \%)(p=0.001)$. We found a significant difference in $G$ allele frequency of TNFAIP3 ( $r 55029939)(37.8 \%$ with SLE vs. $5.6 \%$ with controls, $p=0.001)$. Genotype CG was significantly associated with lupus nephritis and neuropsychiatric manifestations $(p<0.05)$. Although the response to treatment was numerically higher with the genotype $C C$, it did not reach statistical significance $(p=0.4)$.

Conclusion: Our study suggests that TNFAIP3 rs5029939 gene polymorphism is associated with SLE susceptibility and may have an impact on its clinical phenotype. As such association differs among populations of diverse ethnic backgrounds, larger genome-wide association studies are warranted to further elucidate genetic associations.

Keywords: Gene polymorphism, lupus nephritis, systemic lupus erythematosus, TNFAIP3, tumor necrosis factor-alpha inducible protein 3.
\end{abstract}

Systemic lupus erythematosus (SLE) is a systemic autoimmune disease $e^{1,2}$ with an incidence and prevalence varying depending on age, sex, and race. ${ }^{3}$ The highest estimates of incidence and prevalence of SLE were in the North America (23.2/100,000 person-years and 241/100,000 individuals, respectively). ${ }^{4}$ Arab-Americans show a 2.1-fold higher incidence of SLE, compared to non-Arab Caucasians and African Americans, ${ }^{5}$ whereas the incidence and prevalence of SLE in the United Arab Emirates were estimated to be 8.6 per 100,000/year and 103/100,000 individuals (84.5 to 124.4 ), respectively. ${ }^{6}$

Although the exact pathogenesis of SLE still remains to be elucidated, it has been proposed that the interaction of genetic, environmental, and hormonal factors, ultimately leading to the inappropriate function of the innate immune system, altered self-tolerance mechanisms, and apoptotic cell clearance are implicated.' Several genetic polymorphisms have been suggested to predispose to such immune dysregulation,

Received: February 04, 2021 Accepted: April 08, 2021 Published online: October 16, 2021

Correspondence: Reham Abd-elkhalek, MD. Department of Rheumatology and Rehabilitation, Faculty of Medicine, Zagazig University, 44519 Zagazig, Egypt. Tel: 00201016073805 e-mail: ehamabdelkhalek@medicine.zu.edu.eg

\section{Citation:}

Gaballah H, Abd-Elkhalek R, Hussein O, El-Wahab SA. Association of TNFAIP3 gene polymorphism (rs5029939) with susceptibility and clinical phenotype of systemic lupus erythematosus. Arch Rheumatol 2021;36(4):570-576.

(C2021 Turkish League Against Rheumatism. All rights reserved.

This is an open access article under the terms of the Creative Commons Attribution-NonCommercial License, which permits use, distribution and reproduction in any medium, provided the original work is properly cited and is not used for commercial purposes (http://creativecommons.org/licenses/by-nc/4.0/). 
thus influencing SLE susceptibility and clinical features. ${ }^{8,9}$

The tumor necrosis factor-alpha inducible protein 3 (TNFAIP3) gene encodes the ubiquitinmodifying enzyme A20 which inhibits nuclear factor-kappa B (NF-kB) activity in several signaling pathways, including those of TNF and Toll-like receptors. ${ }^{10}$ It is also required for the negative regulation of inflammatory responses. ${ }^{11}$ Over the last decade, numerous studies have investigated the association between TNFAIP3 gene polymorphisms and SLE. ${ }^{12}$ Significant associations have been reported between different TNFAIP3 gene polymorphisms, such as rs2230926, rs5029939, rs5029937, and rs3757173 polymorphisms and SLE susceptibility and clinical manifestations. ${ }^{13-16}$ Such genetic predisposition varies with the ethnic background. In a meta-analysis, rs5029939 and rs3757173 polymorphisms were associated with SLE in Europeans and Asians, while rs2230926 and rs5029937 polymorphisms were associated with SLE in Europeans only. ${ }^{15}$

Currently, there is a limited number of data regarding the impact of TNFAIP3 rs5029939 gene polymorphism among the SLE patients in Egypt. We, therefore, aimed to investigate the association of TNFAIP3 gene polymorphism with SLE susceptibility and clinical manifestations in a cohort of patients with SLE.

\section{PATIENTS AND METHODS}

This case-control study was conducted at Faculty of Medicine, Zagazig University, Department of Rheumatology and Rehabilitation between March 2017 and February 2020. A total of 180 participants (18 males, 72 females; mean age: $30.9 \pm 10.1$ years; range 17 to 59 years) including 90 SLE patients and 90 age- and sexmatched healthy controls recruited from other hospital units and clinics were included. The diagnosis of SLE was made according to the 2012 Systemic Lupus International Collaborating Clinics (SLICC) classification criteria for SLE. ${ }^{17}$ Patients with juvenile SLE ( $<16$ years) or other autoimmune diseases and patients with cardiac, respiratory, neuropsychiatric, and renal diseases due to causes other than SLE were excluded from the study. A written informed consent was obtained from each participant. The study protocol was approved by the Institutional Review Board of the Zagazig University, Faculty of Medicine (No: 3845/17-6-2017, Date: 17.07.2017). The study was conducted in accordance with the principles of the Declaration of Helsinki.

\section{Data collection}

Patients' data were collected from the medical records. Demographic data, clinical manifestations, laboratory and immunological parameters were noted. Disease activity was assessed by the Systemic Lupus Erythematosus Disease Activity Index (SLEDAI) ${ }^{18}$ and severity was assessed by the SLICC and American College of Rheumatology (ACR) Damage Index for SLE. ${ }^{19}$ The response to induction therapy was assessed using the SLE Responder Index (SRI). ${ }^{20}$

\section{Analysis for TNFAIP3 rs5029939 gene polymorphism}

The presence of the TNFAIP3 rs5029939 variant was identified by polymerase chain reaction-restriction fragment length polymorphism (PCR-RFLP). ${ }^{21}$

\section{Deoxyribonucleic acid (DNA) extraction}

Venous blood samples were collected in an ethylenediamine tetraacetic acid (EDTA) tube. The DNA was extracted from peripheral leukocytes using the GeneJET ${ }^{\mathrm{TM}}$ whole blood genomic DNA purification mini kit (Thermo Fisher Scientific ${ }^{\circledR}$ Inc., CA, USA) according to the manufacturer's instructions.

\section{TNFAIP3 rs5029939 variant genotyping}

Amplification was performed in a $50 \mu \mathrm{L}$ reaction volume containing $25 \mu \mathrm{L}$ of $\mathrm{MyTaq}^{\mathrm{TM}}$ Red Mix (Bioline Reagents Ltd., UK) genotyping master mix. The PCR test was carried out using the primer pair 5' GCC TTC ACC AGC AAA TCA AG 3' and 5' GAC ACC AAC TGC AAA GGA GCC AG 3'. The PCR conditions were initial denaturation at $95^{\circ} \mathrm{C}$ for 1 min followed by 35 cycles of $15 \mathrm{sec}$ at $95^{\circ} \mathrm{C}, 15 \mathrm{sec}$ at $50^{\circ} \mathrm{C}, 10 \mathrm{sec}$ at $72^{\circ} \mathrm{C}$, and a final extension at $72^{\circ} \mathrm{C}$ for $5 \mathrm{~min}$. The PCR product (186 bp) was digested with AflII enzyme (New England Biolabs ${ }^{\circledR}$ Inc., MA, USA). Two fragments were produced (142 and $44 \mathrm{bps}$ ) for the single nucleotide polymorphism (SNP) rs5029939 CC genotype, and only one band of 186 bps for the GG genotype. The amplified 
PCR products and digested fragments were visualized by ethidium bromide-stained agarose gel electrophoresis.

\section{Statistical analysis}

Power analysis and sample size calculation were performed using the G*Power version 3.1.9.7 (Heinrich-Heine-Universität Düsseldorf, Düsseldorf, Germany). ${ }^{22}$ The study power was calculated as $95.9 \%$ with an effect (d) size $=0.3$ (depending on difference in minor allele frequency), $\alpha=0.05$, and $\mathrm{df}=2$. A total of 180 participants ( $n=90$ in each group) were needed to be recruited for the study.

Statistical analysis was performed using the IBM SPSS version 20.0 software (IBM Corp., Armonk, NY, USA). Continuous variables were expressed in mean \pm standard deviation (SD) or median (min-max), while categorical variables were expressed in number and frequency. Agreement with Hardy-Weinberg equilibrium
(HWE) was assessed by comparing the expectedto-observed genotype frequencies. The differences in allele and genotype frequencies between cases and controls were analyzed using the Pearson chi-square $\left(\chi^{2}\right)$ test and/or Fisher's exact test. The relative risk (odds ratio) for each genotype and allele was estimated with a 95\% confidence interval (CI). The phenotypic comparisons were done by Pearson $\chi^{2}$ test to compare the frequency of different clinical and laboratory characteristics, as well as the treatment response and SLICC/ACR-Damage Index scores among SLE patients with different genotypes. A $p$ value of $<0.05$ was considered statistically significant.

\section{RESULTS}

Both groups were similar in demographic characteristics including age and sex $(p=0.9$ and

\begin{tabular}{|c|c|c|c|c|c|}
\hline Characteristics & $\mathrm{n}$ & $\%$ & Mean \pm SD & Median & Range \\
\hline Age (year) & & & $30.9 \pm 10.1$ & & \\
\hline \multicolumn{6}{|l|}{ Sex } \\
\hline Male & 18 & 20 & & & \\
\hline Female & 72 & 80 & & & \\
\hline Disease duration (year) & & & & 5 & $1-17$ \\
\hline Fever & 19 & 21.1 & & & \\
\hline Malar rash & 70 & 77.8 & & & \\
\hline Photosensitivity & 60 & 66.7 & & & \\
\hline Oral/nasal ulcers & 43 & 47.8 & & & \\
\hline Discoid lesion & 6 & 6.7 & & & \\
\hline Lupus nephritis & 49 & 54.4 & & & \\
\hline Cardiac & 37 & 41.1 & & & \\
\hline Pulmonary & 44 & 48.9 & & & \\
\hline Neuropsychiatric & 46 & 51.1 & & & \\
\hline GIT & 26 & 28.9 & & & \\
\hline Leucopenia & 26 & 28.9 & & & \\
\hline Anemia & 77 & 85.6 & & & \\
\hline Thrombocytopenia & 7 & 7.8 & & & \\
\hline High ESR & 87 & 96.7 & & & \\
\hline High CRP & 42 & 46.7 & & & \\
\hline Positive ANA & 88 & 97.8 & & & \\
\hline Positive anti-dsDNA & 37 & 41.1 & & & \\
\hline
\end{tabular}




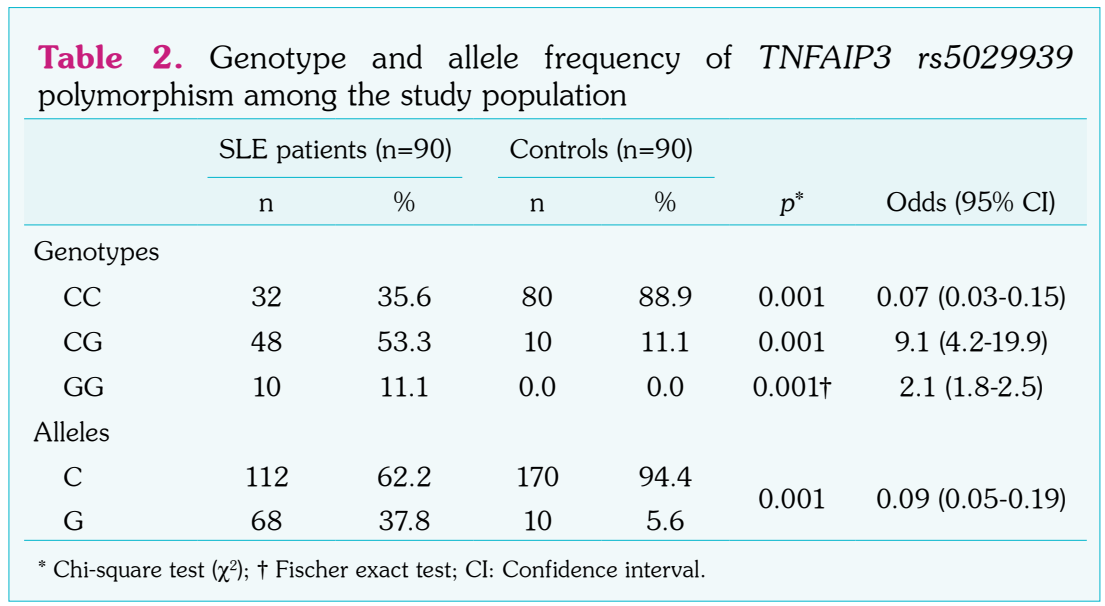

$\mathrm{p}=0.4$, respectively). Demographic and disease characteristics of patients with SLE are shown in Table 1.

We first compared the frequencies of TNFAIB5 rs5029939 genotypes and alleles between the two groups (Table 2). Distribution of the three genotypes differed significantly where genotype CC was more frequent in controls (88.9\%) than
SLE patients (35.6\%), while genotypes CG and GG were more distributed among SLE patients than controls $(53.3 \%$ vs. $11.1 \%$ and $11.1 \%$ vs. $0 \%$, respectively; $(p=0.001)$. Allele distribution also differed significantly between the two groups. The wild allele $\mathrm{C}$ was more frequent in controls (94.4\%) than SLE patients (62.2\%), while the mutant allele $\mathrm{G}$ was more frequent in SLE patients $(37.8 \%)$ than controls (5.6\%).

Table 3. Association between TNFAIP3 rs5029939 polymorphism and the clinical and laboratory characteristics of SLE patients

\begin{tabular}{|c|c|c|c|c|c|c|c|c|}
\hline \multirow[b]{2}{*}{ Manifestations } & \multirow[b]{2}{*}{$\mathrm{n}$} & \multicolumn{2}{|c|}{ Genotype CC $(n=32)$} & \multicolumn{2}{|c|}{ Genotype CG $(n=48)$} & \multicolumn{2}{|c|}{ Genotype GG $(n=10)$} & \multirow[b]{2}{*}{$p^{*}$} \\
\hline & & $\mathrm{n}$ & $\%$ & $\mathrm{n}$ & $\%$ & $\mathrm{n}$ & $\%$ & \\
\hline Fever & 19 & 8 & 42.1 & 11 & 57.9 & 0 & 0.0 & 0.2 \\
\hline Malar rash & 70 & 24 & 34.3 & 36 & 51.4 & 10 & 14.3 & 0.2 \\
\hline Photosensitivity & 60 & 20 & 33.3 & 33 & 55.0 & 7 & 11.7 & 0.8 \\
\hline Oral/nasal ulcers & 43 & 17 & 39.5 & 24 & 55.8 & 2 & 4.7 & 0.16 \\
\hline Discoid lesion & 6 & 4 & 66.7 & 2 & 33.3 & 0 & 0.0 & 0.2 \\
\hline Lupus nephritis & 49 & 19 & 38.9 & 24 & 48.9 & 5 & 12.2 & 0.001 \\
\hline Cardiac & 37 & 9 & 24.3 & 21 & 56.8 & 7 & 18.9 & 0.06 \\
\hline Pulmonary & 44 & 16 & 36.4 & 21 & 47.7 & 7 & 15.9 & 0.3 \\
\hline Neuropsychiatric & 46 & 18 & 39.1 & 28 & 60.9 & 0 & 0.0 & 0.003 \\
\hline GIT & 26 & 7 & 26.9 & 17 & 65.4 & 2 & 7.7 & 0.3 \\
\hline Leucopenia & 26 & 8 & 30.8 & 16 & 61.5 & 2 & 7.7 & 0.6 \\
\hline Anemia & 77 & 25 & 32.5 & 42 & 54.5 & 10 & 13.0 & 0.19 \\
\hline Thrombocytopenia & 7 & 4 & 57.1 & 3 & 42.9 & 0 & 0.0 & 0.3 \\
\hline High ESR & 87 & 32 & 36.8 & 45 & 51.7 & 10 & 11.5 & 0.2 \\
\hline High CRP & 42 & 18 & 42.9 & 22 & 52.4 & 2 & 4.7 & 0.1 \\
\hline Positive ANA & 88 & 30 & 34.1 & 48 & 54.5 & 10 & 11.4 & 0.1 \\
\hline Positive anti-dsDNA & 37 & 9 & 24.3 & 24 & 64.9 & 4 & 10.8 & 0.1 \\
\hline
\end{tabular}

SLE: Systemic lupus erythematosus; GIT: Gastrointestinal tract; ESR: Erythrocyte sedimentation rate; CRP: C-reactive protein; ANA: Antinuclear antibody; Anti-dsDNA: Anti-double-stranded deoxyribonucleic acid; * Chi square test $\left(\chi^{2}\right)$. 
Table 4. Association between TNFAIP3 rs5029939 polymorphism and response to treatment and SLICC/ACRDamage Index in SLE patients

\begin{tabular}{|c|c|c|c|c|c|c|c|c|}
\hline & \multirow[b]{2}{*}{$\mathrm{n}$} & \multicolumn{2}{|c|}{ Genotype CC $(\mathrm{n}=32)$} & \multicolumn{2}{|c|}{ Genotype CG $(n=48)$} & \multicolumn{2}{|c|}{ Genotype GG $(n=10)$} & \multirow[b]{2}{*}{$p^{*}$} \\
\hline & & $\mathrm{n}$ & $\%$ & $\mathrm{n}$ & $\%$ & $\mathrm{n}$ & $\%$ & \\
\hline \multicolumn{9}{|l|}{ Response to therapy } \\
\hline Non-refractory & 49 & 20 & 62.5 & 25 & 52.1 & 4 & 40.0 & \multirow{2}{*}{0.4} \\
\hline Refractory & 41 & 12 & 37.5 & 23 & 47.9 & 6 & 60.0 & \\
\hline \multicolumn{9}{|l|}{ SLICC/ACR-Damage Index } \\
\hline Less severe $\leq$ median (4) & 50 & 19 & 38.0 & 26 & 52.0 & 5 & 10.0 & \multirow{2}{*}{0.8} \\
\hline More severe $>$ median (4) & 40 & 13 & 32.5 & 22 & 55.0 & 5 & 12.5 & \\
\hline
\end{tabular}

SLICC/ACR: Systemic Lupus International Collaborating Clinics/American College of Rheumatology; SLE: Systemic lupus erythematosus; ${ }^{*}$ Chi square test $\left(\chi^{2}\right)$.

Table 5. Comparative analysis between the current study and other studies regarding TNFAIP3 rs5029939 association with lupus susceptibility and phenotype

\begin{tabular}{llcccc}
\hline Studies & Type of the study & Population & Risk allele & $p$ & Phenotypic association* \\
\hline Lodolce et al. ${ }^{27}$ & Candidate gene study & African-Americans, Europeans & G & 0.0453 & Not studied \\
Kim et al. ${ }^{25}$ & Candidate gene study & Koreans & G & 0.01 & Arthritis \\
Elghzaly et al. ${ }^{26}$ & Candidate gene study & Egyptians & G & 0.670 & No significant association \\
Zhang et al. ${ }^{15}$ & Meta-analysis & Europeans, Asians, Africans & G & $4.351 \times 10^{-4}$ & Not studied \\
Current study & Candidate gene study & Egyptians & G & 0.001 & Nephritis \\
& & & & & Neurological \\
${ }^{*} \mathrm{p}<0.05$ for all associations. & & &
\end{tabular}

Subsequently, we examined the association between different genotypes and the clinical manifestations among SLE patients. There was a significant association between genotype CG with lupus nephritis and neuropsychiatric manifestations, while no significant association was evident between the genotypes and all other clinical, laboratory, and immunological parameters (Table 3).

Finally, we assessed the association between genotype frequencies and both SLE response to treatment and ACR-Damage Index. Although the genotype $\mathrm{CC}$ had a higher treatment response rate, it did not reach statistical significance $(p=0.4)$. On the other hand, there was no significant association between the SLICC/ACRDamage Index scores and the different genotypes (Table 4).

\section{DISCUSSION}

Multiple host genetic factors play an important role in both SLE pathogenesis and the development of its clinical features. ${ }^{23,24}$ The TNFAIP3 gene has been suggested to be implicated in B cell activation and autoantibody production related to SLE pathogenesis. Genetic variants in the TNFAIP3 gene may, thus, be suggested as genetic risk factors for SLE susceptibility. ${ }^{25}$ In our study, the SNP, rs5029939, in the TNFAIP3 gene was found to have an association with the risk of SLE and some of its clinical patterns.

Our results also showed that SLE patients differed significantly from the control group in their genotype and allele frequency of TNFAIP3 rs5029939 gene polymorphism. While allele C was dominant in healthy controls, the minor allele $\mathrm{G}$ occurred more frequently in the SLE patients. The genotype CC was the most frequent genotype in healthy controls, while the heterozygous genotype CG, followed by $\mathrm{CC}$, was the most frequent in SLE patients. Results from previous studies assessing the TNFAIP3 rs5029939 and SLE susceptibility and phenotype in different ethnic groups are inconsistent (Table 5). In contrast to our results, a previous study did not 
show a significant association between TNFAIP3 rs5029939 and lupus risk or clinical phenotype, yet the probability of type II statistical error could not be excluded. ${ }^{26}$ However, this SNP (rs5029939) was previously demonstrated to be associated with SLE susceptibility both in the African Americans and Europeans. ${ }^{27} \mathrm{~A}$ similar association between TNFAIP3 rs5029939 gene polymorphism and SLE susceptibility was observed in a Korean study, where the minor allele of TNFAIP3 rs5029939 SNP occurred more frequently in patients with SLE than in the controls. ${ }^{25} \mathrm{~A}$ more recent meta-analysis showed a significant association between the minor rs5029939 allele and SLE susceptibility in the overall population, while, based on the stratification according to ethnicity, rs5029939 was significantly associated with SLE in Europeans, but not in Asians or Africans. ${ }^{15}$ These findings indicate that genetic predisposition is not uniform among different ethnic groups, but rather affected by the interplay of multiple host factors.

The clinical manifestations and disease course vary greatly among lupus patients. Such clinical heterogeneity can be correlated with specific polymorphisms in SLE-associated genes. ${ }^{28}$ Our results suggest an impact of TNFAIP3 rs5029939 gene polymorphism on disease phenotype among our lupus population. There was a significant association between genotype GC with lupus nephritis and neuropsychiatric manifestations. In the Korean SLE population, the arthritis phenotype was marginally associated with TNFAIP3 SNPs; however, no association was found with the presence of nephritis. ${ }^{25}$ Another study involving Caucasian SLE patients suggested that the risk of developing renal or hematological manifestations almost doubled in SLE patients with the heterozygous risk allele of TNFAIP3 rs5029939, compared to homozygous non-risk subjects. ${ }^{13}$ However, in a Chinese study, no significant association was identified between TNFAIP3 gene polymorphisms and any of the studied clinical, hematological, or immunological parameters. ${ }^{12}$

Our study did not show a significant difference in SLICC/ACR-Damage Index scores among SLE patients of different genotypes. The treatment response rate was numerically higher with the genotype CC, but not statistically significant. Of note, SLE is an attractive candidate for a personalized immunotherapy approach, since individual patients may have different molecular pathways driving their disease. ${ }^{29}$ The TNFAIP3 can be considered a central regulator of immunopathology in SLE, as it acts at multiple steps in the NF- $\mathrm{KB}$ signaling pathway. ${ }^{30}$ Based on our findings, we can recommend future studies to investigate therapies targeting the TNFAIP3 pathway in patients with nephritis and neuropsychiatric SLE. This may represent an interesting potential therapeutic target in a subset of lupus patients.

The present study is limited by its singlecenter nature and relatively small sample size, thus hindering data generalization. However, it indicates the impact of genetic polymorphism not only on disease susceptibility, but also on its clinical phenotype among patients with SLE in our country.

In conclusion, our study suggests that TNFAIP3 rs5029939 gene polymorphism is associated with the development of SLE and may have an impact on its clinical phenotype. Such an association, however, differs from previous studies involving population of other ethnic backgrounds. Therefore, further multi-center, genome-wide association studies are needed to further elucidate different genetic associations and their interactions in a larger population.

\section{Declaration of conflicting interests}

The authors declared no conflicts of interest with respect to the authorship and/or publication of this article.

\section{Funding}

The authors received no financial support for the research and/or authorship of this article.

\section{REFERENCES}

1. Justiz Vaillant AA, Goyal A, Bansal P, Varacallo M. Systemic Lupus Erythematosus. 2020 Dec 30. In: StatPearls [Internet]. Treasure Island (FL): StatPearls Publishing; 2021

2. Tanaka Y. State-of-the-art treatment of systemic lupus erythematosus. Int J Rheum Dis 2020;23:465-71.

3. Stojan G, Petri M. Epidemiology of systemic lupus erythematosus: An update. Curr Opin Rheumatol 2018;30:144-50.

4. Rees F, Doherty M, Grainge MJ, Lanyon P, Zhang $\mathrm{W}$. The worldwide incidence and prevalence of 
systemic lupus erythematosus: A systematic review of epidemiological studies. Rheumatology (Oxford) 2017;56:1945-61.

5. Housey M, DeGuire P, Lyon-Callo S, Wang L, Marder $\mathrm{W}$, McCune WJ, et al. Incidence and prevalence of systemic lupus erythematosus among Arab and Chaldean Americans in southeastern Michigan: The Michigan Lupus Epidemiology and Surveillance Program. Am J Public Health 2015;105:e74-9.

6. Al Dhanhani AM, Agarwal M, Othman YS, Bakoush $\mathrm{O}$. Incidence and prevalence of systemic lupus erythematosus among the native Arab population in UAE. Lupus 2017;26:664-9.

7. Moulton VR, Suarez-Fueyo A, Meidan E, Li H, Mizui M, Tsokos GC. Pathogenesis of human systemic lupus erythematosus: A cellular perspective. Trends Mol Med 2017;23:615-35.

8. Sun C, Molineros JE, Looger LL, Zhou XJ, Kim $\mathrm{K}$, Okada Y, et al. High-density genotyping of immune-related loci identifies new SLE risk variants in individuals with Asian ancestry. Nat Genet 2016;48:323-30.

9. Ramos PS, Brown EE, Kimberly RP, Langefeld CD. Genetic factors predisposing to systemic lupus erythematosus and lupus nephritis. Semin Nephrol 2010;30:164-76.

10. Das T, Chen Z, Hendriks RW, Kool M. A20/ Tumor necrosis factor $\alpha$-induced protein 3 in immune cells controls development of autoinflammation and autoimmunity: Lessons from mouse models. Front Immunol 2018;9:104.

11. Zhong J, Shi G. Editorial: Regulation of inflammation in chronic disease. Front Immunol 2019;10:737.

12. Han JW, Wang Y, Li HB, Alateng C, Bai YH, Sun ZQ, et al. Single nucleotide polymorphisms of TNFAIP3 are associated with systemic lupus erythematosus in Han Chinese population. Int J Immunogenet 2016;43:96-100.

13. Bates JS, Lessard CJ, Leon JM, Nguyen T, Battiest LJ, Rodgers $\mathrm{J}$, et al. Meta-analysis and imputation identifies a $109 \mathrm{~kb}$ risk haplotype spanning TNFAIP3 associated with lupus nephritis and hematologic manifestations. Genes Immun 2009;10:470-7.

14. Lee YH, Song GG. Associations between TNFAIP3 gene polymorphisms and systemic lupus erythematosus: A meta-analysis. Genet Test Mol Biomarkers 2012;16:1105-10.

15. Zhang MY, Yang XK, Pan HF, Ye DQ. Associations between TNFAIP3 gene polymorphisms and systemic lupus erythematosus risk: An updated meta-analysis. HLA 2016;88:245-52.

16. Ciccacci C, Latini A, Perricone C, Conigliaro P, Colafrancesco S, Ceccarelli F, et al. TNFAIP3 gene polymorphisms in three common autoimmune diseases: Systemic lupus erythematosus, rheumatoid arthritis, and primary Sjogren syndromeassociation with disease susceptibility and clinical phenotypes in Italian patients. J Immunol Res 2019;2019:6728694.
17. Petri M, Orbai AM, Alarcón GS, Gordon C, Merrill JT, Fortin PR, et al. Derivation and validation of the Systemic Lupus International Collaborating Clinics classification criteria for systemic lupus erythematosus. Arthritis Rheum 2012;64:2677-86.

18. Gladman DD, Ibañez D, Urowitz MB. Systemic lupus erythematosus disease activity index 2000. J Rheumatol 2002;29:288-91.

19. Gladman DD, Urowitz MB, Goldsmith $\mathrm{CH}$, Fortin $\mathrm{P}$, Ginzler $\mathrm{E}$, Gordon $\mathrm{C}$, et al. The reliability of the Systemic Lupus International Collaborating Clinics/ American College of Rheumatology Damage Index in patients with systemic lupus erythematosus. Arthritis Rheum 1997;40:809-13.

20. Furie RA, Petri MA, Wallace DJ, Ginzler EM, Merrill JT, Stohl W, et al. Novel evidence-based systemic lupus erythematosus responder index. Arthritis Rheum 2009;61:1143-51.

21. Yang HW, Xie Y, Zhao Y, Sun L, Zhu X, Wang S, et al. TNFAIP3 gene rs7749323 polymorphism is associated with late onset myasthenia gravis. Medicine (Baltimore) 2017;96:e6798.

22. Faul F, Erdfelder E, Lang AG, Buchner A. G*Power 3: A flexible statistical power analysis program for the social, behavioral, and biomedical sciences. Behav Res Methods 2007;39:175-91.

23. Mu Q, Zhang H, Luo XM. SLE: Another autoimmune disorder influenced by microbes and diet? Front Immunol 2015;6:608.

24. Barcia-Sixto L, Isenberg D. Systemic lupus erythematosus: Causes and manifestations. Trends Urology \& Men Health 2020;11:26-9.

25. Kim SK, Choe JY, Bae J, Chae SC, Park DJ, Kwak SG, et al. TNFAIP3 gene polymorphisms associated with differential susceptibility to rheumatoid arthritis and systemic lupus erythematosus in the Korean population. Rheumatology (Oxford) 2014;53:1009-13.

26. Elghzaly AA, Metwally SS, El-Chennawi FA, Elgayaar MA, Mosaad YM, El-Toraby EE, et al. IRF5, PTPN22, CD28, IL2RA, KIF5A, BLK and TNFAIP3 genes polymorphisms and lupus susceptibility in a cohort from the Egypt Delta; relation to other ethnic groups. Hum Immunol 2015;76:525-31.

27. Lodolce JP, Kolodziej LE, Rhee L, Kariuki SN, Franek BS, McGreal NM, et al. African-derived genetic polymorphisms in TNFAIP3 mediate risk for autoimmunity. J Immunol 2010;184:7001-9.

28. Joo YB, Lim J, Tsao BP, Nath SK, Kim K, Bae SC. Genetic variants in systemic lupus erythematosus susceptibility loci, XKR6 and GLT1D1 are associated with childhood-onset SLE in a Korean cohort. Sci Rep 2018;8:9962.

29. McGlasson S, Wiseman S, Wardlaw J, Dhaun N, Hunt DPJ. Neurological disease in lupus: Toward a personalized medicine approach. Front Immunol 2018;9:1146.

30. Vereecke L, Beyaert R, van Loo G. The ubiquitinediting enzyme A20 (TNFAIP3) is a central regulator of immunopathology. Trends Immunol 2009;30:383-91. 